\title{
HOW TO DEAL WITH MULTI-SOURCE DATA FOR TREE DETECTION BASED ON DEEP LEARNING
}

\author{
Lionel Pibre ${ }^{a, e}$, Marc Chaumont ${ }^{a, b}$, Gérard Subsol ${ }^{a, c}$, Dino Ienco ${ }^{d}$ and Mustapha Derras ${ }^{e}$ \\ ${ }^{a}$ LIRMM laboratory, University of Montpellier, Montpellier, France; ${ }^{b}$ University of Nîmes, Nîmes, France; \\ ${ }^{\mathrm{c}}$ CNRS; ${ }^{\mathrm{d}}$ IRSTEA; ${ }^{\mathrm{e}}$ Berger-Levrault, France
}

\begin{abstract}
In the field of remote sensing, it is very common to use data from several sensors in order to make classification or segmentation. Most of the standard Remote Sensing analysis use machine learning methods based on image descriptions as HOG or SIFT and a classifier as SVM. In recent years neural networks have emerged as a key tool regarding the detection of objects. Due to the heterogeneity of information (optical, infrared, LiDAR), the combination of multi-source data is still an open issue in the Remote Sensing field. In this paper, we focus on managing data from multiple sources for the task of localization of urban trees in multi-source (optical, infrared, DSM) aerial images and we evaluate the different effects of preprocessing on the input data of a CNN.
\end{abstract}

Index Terms - Deep Learning, Localization, Multisource Data, Data Fusion, Remote Sensing

\section{INTRODUCTION}

Nowadays, it is common to combine different information sources in order to deal with the object detection task [1] and more particularly in the field of remote sensing [2]. Indeed, it is admitted that the "heterogeneity" of remote sensing information (optical, near-infrared, LiDAR) can improve the object detection.

In the case of multi-source data (optical, infrared, LiDAR), it is nevertheless complex to merge several information sources since they provide measurements that can be different and complementary in their nature [3]. It is therefore crucial considering the integration issue during the conception of an object detection method since, the way in which different data are combined can drastically impact the final result.

Recently, the Deep Learning [4] methods have shown that neural network models, and more specifically Convolutional Neural Networks (CNNs) are tailored to image classification [5] and localization [6]. CNNs [7] integrate in a single optimization schema both the learning of a classification model and the learning of a suitable set of descriptors of images.

CONTACT Lionel Pibre. Email:lionel.pibre@lirmm.fr
In this paper, we address the specific problem of localization and detection of urban trees in multi-source aerial data composed of synchronized optical, near infrared and Digital Surface Model (DSM) measurements of urban areas. We should notify the reader that we are looking to localize each tree in the image. This task is more complicated than a simple global classification of an image. The task is also difficult due to the overlapping between trees. The task is an object detection and not a pixel labelling which is also more complicated.

The current approaches in the remote sensing field are often ad hoc or heuristic [2]. In this paper, we propose to use CNN which indeed give a better solution. If we are looking for a more generalized problem of object detection, the solution is using CNN [8] but usually only with RGB images (and not near-infrared and/or DSM images) and the objects are often not overlapping or close.

Furthermore, we want to emphasize the importance of the image preprocessing when using a Deep Learning approach. Indeed, we try to demystify the CNNs by showing that the preparation of the learning data is of paramount importance on the performances of the CNNs.

The rest of the paper is organized as follows: Section 2 introduces CNNs and specifies the network architecture, Section 3 describes our approach. Experimental setting and results are discussed in Section 4. Conclusions are drawn in Section 5.

\section{DEEP LEARNING PRELIMINARIES}

A neural network [4] is a mathematical model whose design is inspired by the biological neurons. Initially, they were proposed to model the behavior of a brain. Since the 90's, they have been used in Artificial Intelligence for learning purpose. Moreover, challenges such as ImageNet showed that these approaches reached high classification performances $[5,9]$.

Neural networks are composed of different layers. The first layer is called the input layer, this layer is fed by the original data. The intermediate layers are called hidden layers and finally there is the output layer which returns the prediction. All these layers are composed of neurons that perform operations on their input values (see Equation (1)). 


$$
\sigma_{k}^{(l)}=\mathbf{x}_{k}^{(l-1)} \cdot \mathbf{w}_{k}^{(l)}+b_{k}^{(l)}
$$

where $\sigma_{k}^{(l)} \in \mathbb{R}$ is the result of the $l-1$ layer, $\mathbf{x}_{k}^{(l-1)} \in \mathbb{R}$ are the neuron outputs coming from the $l-1$ layer with $k=$ $\left\{1, \ldots, K^{(l-1)}\right\}$ and $\mathbf{w}_{k}^{(l)} \in \mathbb{R}$ are the weights.

In a convolutional neural network, hidden layers are composed of three successive processing: the convolution, the application of an activation function and finally the pooling.

The convolution of the first layer is a classical convolution. The remaining convolutions are somewhat more specific since the resulting images of these convolutions are the sums of $K^{(l-1)}$ convolutions, where $K^{(l-1)}$ is the number of outputs of the $l-1$ layer.

After the convolution, a non-linear function called activation function is applied to each value of the filtered image. The activation function may be a Gaussian function: $f(x)=e^{-\frac{x^{2}}{\sigma^{2}}}$ a ReLU [10] (Rectified Linear Unit): $f(x)=$ $\max (0, x)$, etc... These functions allow to break the linearity related to the convolutions. The pooling is an aggregation operation which reduces the dimension of the feature maps, and allows to reduce the number of calculations. This step is specific to convolutional neural networks. The two common methods employed to perform this operation are: i) the computation of the average (avg-pooling) ii) the selection of the maximum value among a local neighborhood (max-pooling). In addition, in an object classification task, the use of maxpooling allows a translation invariance of the features.

During the last decade, many network architectures have emerged. Among these, some networks have become popular. They have become references of the state of the art. We present here one of these networks, AlexNet [9].

AlexNet [9] appears in 2012 during the ImageNet challenge ${ }^{1}$. This network allowed Krizhevsky et al. to achieve the best performance on the ImageNet database. It consists of five convolutional layers. Each convolutional layer is followed by a ReLU [10] activation function and a max-pooling operation.

\section{PROPOSITION}

In order to locate the trees on the tested images, we used a multi-scale sliding window [11]. Since trees do not have the same crown diameter, this method allows us to detect all trees independently of the size.

As thumbnails images extracted from the sliding window must be of the same size than the images employed during the training phase, instead of varying the size of the sliding window, we vary the size of the tested images. We resize each image from $30 \%$ up to $300 \%$ of their original size with a step of $10 \%$. The sliding window scans the tested images at every scale. Each thumbnail retrieved from the sliding window is given to the network which outputs the probability to contain

\footnotetext{
${ }^{1}$ http://www.image-net.org
}

a tree. The network allows us to determine the area in the entire image that have a high probability of containing a tree.

Applying our sliding window on the same image but at different scales will create an accumulation of bounding boxes over the same area. To overcome this problem we apply a fusion strategy on the set of overlapping bounding boxes classified as a tree. On all bounding boxes that overlap, we apply a strategy of fusion by area $[12,13]$.

The area fusion will compare all the pairs of bounding boxes. For each pair, we compute if one of the two bounding boxes overlaps each other by a percentage bigger than $80 \%$ (see equation (2)). If this is the case, the bounding box with the lowest probability of containing a tree is deleted.

$$
\frac{\operatorname{Area}(B 1 \cap B 2)}{\min (\operatorname{Area}(B 1), \operatorname{Area}(B 2))}>0.8
$$

with $B 1$ and $B 2$ two bounding boxes given by the network.

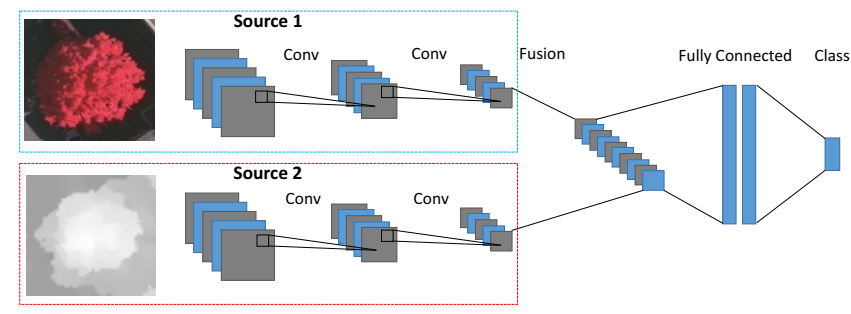

Fig. 1. Example of Late Fusion architecture.

We also used the architecture proposed in [14]. The main idea of this architecture is to treat the different information independently. This kind of architecture is called Late Fusion, an example is given in Figure 1. To construct this architecture, we used the well known AlexNet [9]. As seen in Figure 1, we duplicated the convolutional layers, and we concatenated the two branches before the fully connected layers. We can observe from Figure 1 that the network has two inputs. Each entry corresponds to a different data type.

Moreover, we also used the Normalized Difference Vegetation Index (NDVI) $[15,16]$. This vegetation index is widely applied in the field of remote sensing [2]. This index allows to extract vegetation in images. The NDVI is a non-linear combination of red and near-infrared channels, see Equation 3.

$$
N D V I=\frac{N I R-R e d}{N I R+R e d}
$$

where Red and NIR stand for the spectral reflectance measurements acquired in the visible (red) and near-infrared regions, respectively.

We can observe Figure 2 that the NDVI allows us to remove areas that are not vegetation. Thus, we can easily decrease the number of false positives. Indeed, the different objects that have a shape similar to that of a tree that are not vegetation are no longer present on the NDVI. 


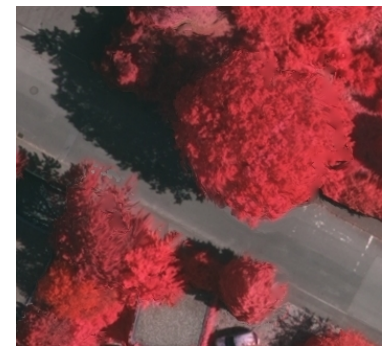

(a) Red, Green, Near-infrared

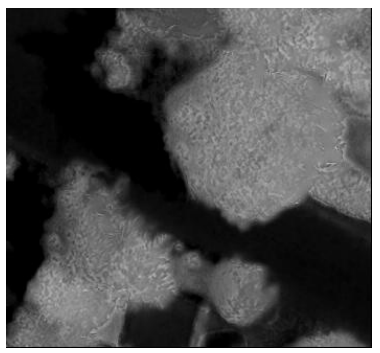

(b) NDVI
Fig. 2. Generated NDVI from an Vaihingen dataset image.

\section{EXPERIMENTAL RESULTS}

In this section we report the experimental settings and we discuss the results we obtained on the Vaihingen dataset. This dataset was captured over Vaihingen city in Germany ${ }^{2}$.

The RGNIR data were acquired using an Intergraph / ZI DMC flying $900 \mathrm{~m}$ height above the ground with $9 \mathrm{~cm}$ ground resolution by the company RWE Power on 24 July and $6 \mathrm{Au}-$ gust 2008 and a Digital Surface Model (DSM) with $9 \mathrm{~cm}$ ground resolution was interpolated from the AirBorne Laser Scanner point cloud acquired on 21 August 2008 by Leica Geosystems using a Leica ALS50 system with 45 field of view and a mean flying height above ground of $500 \mathrm{~m}$.

\subsection{Experimental Settings}

To train ours models we used a training base composed of about 6,000 "tree" thumbnails and 40,000 "other" thumbnails. The thumbnail size is $64 \times 64$ pixels. The thumbnail images of the class "tree" are obtained by manual labeling of 19 entire images while the class "other" is obtained by randomly cropped images (that are not trees) of the 19 annotated images. In addition, to increase the number of thumbnails of the class "tree" (about 1,500 before transformation), we applied rotations of $90^{\circ}, 180^{\circ}$ and $270^{\circ}$ to the thumbnails. Our test base is composed of about twenty images of variable size (from $125 \times 150$ pixels up to $550 \times 725$ pixels) and contains about a hundred trees.

To assess the results, we compute the overlap ratio between the detected bounding box and the ground truth Eq. 4.

$$
\text { label }=\left\{\begin{array}{ccc}
1 & \text { If } & \frac{\text { area }(\text { detection } \cap \text { ground truth })}{\text { area }(\text { detection } \cup \text { ground } \text { truth })}>0.5 \\
0 & \text { If } & \frac{\text { area }(\text { detection } \cap \text { ground truth })}{\text { area }(\text { detection } \cup \text { ground } \text { truth })} \leq 0.5
\end{array}\right.
$$

detection $\cap$ ground truth is the intersection between the detection and the ground truth, and area(detection $\cup$ ground $t r u t h)$ is the union of their area.

\footnotetext{
${ }^{2}$ The Vaihingen data set was provided by the German Society for Photogrammetry, Remote Sensing and Geoinformation (DGPF) [17]: http://www.ifp.uni-stuttgart.de/dgpf/DKEP-Allg.html.
}

First, we tested a mono-band classification (only one source is used for the classification) but also by concatenating the R, G and NIR channels. These tests allow us to see among these different sources, which one is the most interesting to discriminate trees when used alone. Then we compare different ways of integrating data from different sources into a CNN.

For the first way of integrating data from different sources, we have concatenated the different sources (EF Data1/ Data2), this kind of architecture is called Early Fusion (EF). We tested by concatenating the images RGNIR and DSM and finally we concatenated NDVI and DSM. For the last way of integrating data from different sources, we used the Late Fusion architecture described Section 3 (LF Data1/Data2). For these experiments we used the same pairs of data as for the Early Fusion architecture.

We want to remind the reader that our main objective is to show the impact and importance of data preparation when doing Deep Learning. To validate our experimentation, we perform a 5-fold cross validation.

\subsection{Results and Discussions}

Table 1, Table 2 and Table 3 summarize the results we have obtained on the Vaihingen dataset. They depict the average values of Recall, Precision and F-Measure max $_{\text {-Mor }}$ fo different models. We chose to take the point of the recall/precision curve where the f-measure is the highest.

\subsubsection{Limits of using one only source}

Table 1 shows the results we obtained using only one source. As can be seen the results between the different tests are very close. NDVI allows us to obtain the best performance in

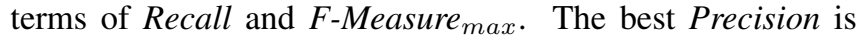
obtained using the DSM. It can be observed that the best results in terms of F-Measure $\max$ are obtained when we have transformed the original data, i.e. when we use the NDVI.

Table 1. Results using one source.

\begin{tabular}{|c|c|c|c|}
\hline Source & RGNIR & DSM & NDVI \\
\hline F-Measure $\max$ & $60.45 \%$ & $62.47 \%$ & $\mathbf{6 3 . 9 7 \%}$ \\
Recall & $57.89 \%$ & $57.62 \%$ & $\mathbf{6 2 . 3 4 \%}$ \\
Precision & $63.44 \%$ & $\mathbf{6 8 . 5 6 \%}$ & $67.04 \%$ \\
\hline
\end{tabular}

\subsubsection{What is the best fusion process?}

Table 2 shows the results we obtained using the Early Fusion. Here, we can see that the best results are obtained when ) using NDVI and DSM, we obtain a F-Measure max $_{\text {max }} 75 \%$. However, when we use RGNIR, the results are lower $(67 \%)$.

Table 3 shows the results that we have obtained using the Late Fusion architecture described in Section 3. The observation is the same as in the previous experiments, better 
Table 2. Results using multi-source data and the concatenation.

\begin{tabular}{|c|c|c|}
\hline EF & RGNIR/DSM & NDVI/DSM \\
\hline F-Measure $\max$ & $67.12 \%$ & $\mathbf{7 5 . 3 0 \%}$ \\
Recall & $65.40 \%$ & $\mathbf{6 8 . 3 7 \%}$ \\
Precision & $69.54 \%$ & $\mathbf{8 4 . 1 1 \%}$ \\
\hline
\end{tabular}

results are obtained when NDVI and DSM are used. When using NDVI and DSM, we obtain an F-Measure max $_{\max }$ of $72 \%$ against $62 \%$ when using RGNIR and DSM. It can also be noted that this architecture gives lesser performances compared to the Early Fusion architecture.

Table 3. Results using multi-source data and the Late Fusion architecture.

\begin{tabular}{|c|c|c|}
\hline LF & RGNIR/DSM & NDVI/DSM \\
\hline F-Measure max $_{\text {max }}$ & $62.14 \%$ & $\mathbf{7 2 . 5 7 \%}$ \\
Recall $^{\text {Precision }}$ & $62.54 \%$ & $\mathbf{7 0 . 9 9 \%}$ \\
Prion & $62.65 \%$ & $\mathbf{7 4 . 8 3 \%}$ \\
\hline
\end{tabular}

The NDVI allows to keep only the essential information. Indeed the NDVI allows us to keep only the vegetation present in the image. This shows us the benefit of using a source which allows us to better discriminate trees, even if this source come from a heuristic transformation. In this case, it is possible that the lack of data prevents the networks to mix incorrectly the data.

Furthermore, we can observe that using DSM with NDVI gives much better results than just use the NDVI. Indeed, both are very important to detect and locate trees. If we detect an object that has a certain height and is vegetation, then there is a great chance that it is a tree. These two pieces of information are really crucial for detecting trees.

It can be noted that when using RGNIR alone, the result is not so far from those of NDVI or DSM. However, when we combine RGNIR and DSM the gain is not significant. Indeed, since these data are very different (their scale of value for example), it is very difficult for the $\mathrm{CNN}$ to discriminate trees correctly by concatenating these two types of data.

Moreover, we can observe that when we use the Late Fusion architecture the results are always inferior to the concatenation. Indeed, when we use RGNIR and DSM the result goes from $62 \%$ to $67 \%$ while using the Early Fusion architecture. Similarly, when tested with NDVI and DSM, the results go from $72 \%$ with the Late Fusion architecture to $75 \%$ when using the Early Fusion architecture. Figure 3 shows two results that we obtained.

\subsubsection{How to select the sources to fuse?}

Furthermore, we computed the correlation between each source using the Jaccard index. We compute the intersection of trees found in two sources over the union of trees found in both sources. The results are presented Table 4 . The second row of Table 4 represents the distribution of trees present only in one source.

Table 4. Results of the correlation between each source.

\begin{tabular}{|c|c|c|c|c|}
\hline Sources & \multicolumn{2}{|c|}{ RGNIR/DSM } & \multicolumn{2}{c|}{ NDVI/DSM } \\
\hline Correlation & \multicolumn{2}{|c|}{$47.86 \%$} & \multicolumn{2}{|c|}{$48.96 \%$} \\
\hline Distribution & $26.47 \%$ & $25.66 \%$ & $28.75 \%$ & $22.27 \%$ \\
\hline
\end{tabular}

We can observe that all the correlations are around 50\%. These results show that among all the trees found, about 50\% of the trees are found in both sources and therefore the remaining 50\% are found in either the first or the second source. Moreover, the second row of the table shows that the remaining $50 \%$ is distributed in the two sources and thus shows us the utility of combining several sources.

We also computed the correlation of false positives between the different sources and we noticed that this correlation never exceeds $10 \%$ regardless of the sources studied. Thus, combining sources should reduce the number of false positives.
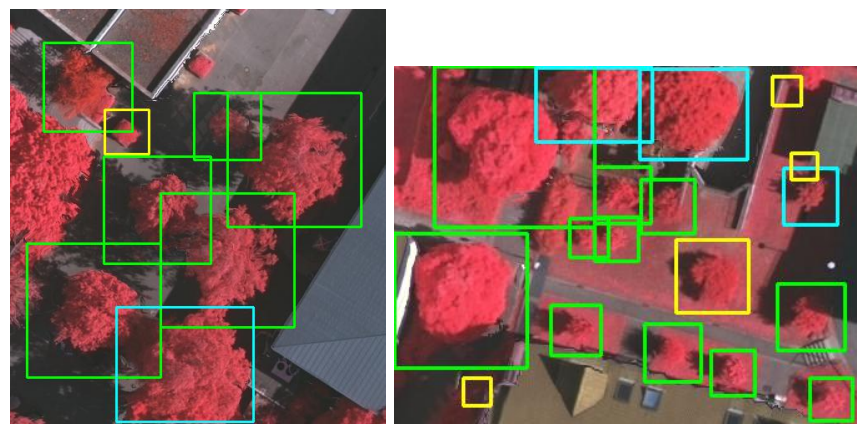

Fig. 3. Examples of the obtained results, in green we have the trees correctly localized, in blue the false negatives and in yellow the false positives.

\section{CONCLUSION}

In this paper, we have evaluated the use of Deep Learning methods to deal with multi-source data (optical, near-infrared and DSM). In addition, we have evaluated the impact of transformations applied to the input data of a CNN. We used the NDVI instead of using the data with the Red, Green and NearInfrared channels. We realized our experiments on a problem of detection and localization of urban trees in multi-source aerial data.

Our work has shown that the use of NDVI allows to obtain the best performances and thus highlights the importance of the data that are used to learn a model with a CNN.

The results we have obtained set a milestone by showing the effectiveness of CNNs in merging different information with a performance gain exceeding $10 \%$. 


\section{REFERENCES}

[1] X. Liu and Y. Bo, "Object-based crop species classification based on the combination of airborne hyperspectral images and lidar data," Remote Sensing, vol. 7, no. 1, pp. 922-950, 2015.

[2] M. Alonzo, B. Bookhagen, and D.A. Roberts, "Urban tree species mapping using hyperspectral and lidar data fusion," Remote Sensing of Environment, vol. 148, pp. 70-83, 2014.

[3] M. Schmitt and X.X. Zhu, "Data fusion and remote sensing: An ever-growing relationship," IEEE Geoscience and Remote Sensing Magazine, vol. 4, no. 4, pp. 6-23, 2016.

[4] Y. LeCun, Y. Bengio, and G.E. Hinton, "Deep learning," Nature, vol. 52, no. 8, pp. 436-444, 2015.

[5] C. Szegedy, W. Liu, Y. Jia, P. Sermanet, et al., "Going deeper with convolutions," in IEEE Conference on Computer Vision and Pattern Recognition, 2015, pp. 19.

[6] T. H. N. Le, Y. Zheng, C. Zhu, K. Luu, and M. Savvides, "Multiple scale faster-rcnn approach to driver's cell-phone usage and hands on steering wheel detection," in 2016 IEEE Conference on Computer Vision and Pattern Recognition Workshops (CVPRW), June 2016, pp. 46-53.

[7] Y. LeCun, L.D. Jackel, L. Bottou, C. Cortes, J. S Denker, H. Drucker, I. Guyon, UA. Muller, E. Sackinger, P. Simard, et al., "Learning algorithms for classification: A comparison on handwritten digit recognition," Neural networks: the statistical mechanics perspective, vol. 261, pp. 276, 1995.

[8] J.H. Bappy and A.K. Roy-Chowdhury, "Cnn based region proposals for efficient object detection," in International Conference on Image Processing (ICIP), 2016 IEEE International Conference on, Phoenix, Arizona, USA, September 2016, IEEE, pp. 3658-3662.

[9] A. Krizhevský, I. Sutskever, and G.E. Hinton, "Imagenet classification with deep convolutional neural networks," in Conference on Neural Information Processing Systems, 2012, pp. 1097-1105.

[10] V. Nair and G.E. Hinton, "Rectified linear units improve restricted boltzmann machines," in International Conference on Machine Learning, 2010, pp. 807-814.

[11] C. Garcia and M. Delakis, "Convolutional face finder: A neural architecture for fast and robust face detection," IEEE Trans. on Pattern Analysis and Machine Intelligence, vol. 26, no. 11, pp. 1408-1423, 2004.
[12] P. Sermanet, D. Eigen, X. Zhang, M. Mathieu, R. Fergus, and Y. LeCun, "Overfeat: Integrated recognition, localization and detection using convolutional networks," in International Conference on Learning Representations (ICLR), Banff, Canada, April 2014.

[13] M. Bertozzi, E. Binelli, A. Broggi, and MD. Rose, "Stereo vision-based approaches for pedestrian detection," in IEEE Conference on Computer Vision and Pattern Recognition (CVPR), 2005, pp. 16-16.

[14] J. Wagner, V. Fischer, M. Herman, and S. Behnke, "Multispectral pedestrian detection using deep fusion convolutional neural networks," in European Symp. on Artificial Neural Networks (ESANN), Bruges, Belgium, April 2016.

[15] G.E Meyer, "Machine vision identification of plants," Recent Trends for Enhancing the Diversity and Quality of Soybean Products. Krezhova D (ed.) Croatia: InTech, 2011.

[16] A. Bannari, D-C. He, D. Morin, and H. Anys, "Analyse de l'apport de deux indices de végétation à la classification dans les milieux hétérogènes," Canadian journal of remote sensing, vol. 24, no. 3, pp. 233-239, 1998.

[17] M. Cramer, "The dgpf-test on digital airborne camera evaluation-overview and test design," Photogrammetrie-Fernerkundung-Geoinformation, vol. 2010, no. 2, pp. 73-82, 2010. 